\title{
OPEN Sarcopenia risk and associated factors among Chinese community-dwelling older adults living alone
}

\author{
Li Cheng ${ }^{1}$, Janet W. H. Sit ${ }^{2}$, Helen Y. L. Chan ${ }^{2}$, Kai Chow Choi ${ }^{2}$, Regina K. Y. Cheung ${ }^{3}$, \\ Martin M. H. Wong ${ }^{2}$, Francis Y. K. Li ${ }^{3}$, Tin Yan Lee ${ }^{3}$, Elina S. M. Fung ${ }^{3}$, Keen Man Tai ${ }^{3}$ \& \\ Winnie K. W. So ${ }^{2 \bowtie}$
}

Sarcopenia, defined as a progressive loss of muscle mass and reduced muscle strength and functional capacity, is common among older adults. This study aimed to assess the proportion of people at risk of sarcopenia and probable sarcopenia among Chinese community-dwelling older adults living alone and to identify the associated factors. A total of 390 older adults were included in this study. Sarcopenia and probable sarcopenia were defined according to the criteria of the Asian Working Group for Sarcopenia 2019. Data on socio-demographic characteristics, health status, health behaviours and lifestyle characteristics, nutritional status, physical activity level, and depressive symptoms were collected. The association between these characteristics and sarcopenia risk was analysed using a multivariate ordinal logistic regression. The proportion of subjects at risk of sarcopenia and probable sarcopenia was found to be $57.7 \%$ and $30 \%$, respectively. Older age, being malnourished and being at risk of malnutrition were significantly associated with sarcopenia risk. Being educated to secondary level or above, being overweight or obese and higher physical activity level were associated with decreased sarcopenia risk. Our results showed that older adults living alone were at high risk of developing sarcopenia and probable sarcopenia. These results emphasise the urgent need to initiate aggressive screening and holistic lifestyle therapeutic intervention strategies for this high-risk population.

Sarcopenia is characterised by age-related decreased skeletal muscle mass, diminished muscle strength, and deteriorated physical performance ${ }^{1}$. It is considered a reliable predictor of poorer prognoses among older adults, and is associated with functional impairment, increased risk of cardiometabolic diseases and falls and fractures, limited mobility, and poor quality of life ${ }^{1,2}$. Sarcopenia also increases the risk of institutionalisation and hospitalisation and significantly increases healthcare expenditure. Sarcopenia, however, is often underdetected and undertreated in mainstream practice. Consensus updates of both the European Working Group on Sarcopenia in Older People (EWGSOP2) and the Asian Working Group for Sarcopenia (AWGS) 2019 highlighted the importance of developing methods for the early identification of those at risk for sarcopenia in the community setting without advanced diagnostic tests ${ }^{1,3}$. Consensus updates also introduced the term 'probable sarcopenia', defined as low muscle strength with or without reduced physical performance. In view of the reversible nature of this condition, early detection of older adults with probable sarcopenia could facilitate timely education, implementation of lifestyle interventions, and referral for confirmatory diagnosis.

The increasing number of older adults living alone is a growing global challenge. Owing to the recent improvements in the housing quality, living alone may have become a preferred alternative living arrangement for older adults, but it may often be an inevitable arrangement when they become single, divorced, or widowed, or having no informal caregivers looking after them. Living alone is usually accompanied with feelings of loneliness and insecurity and difficulties in daily living, all of which pose enormous challenges for older adults ${ }^{4}$. Evidence consistently supports that living alone is associated with increased risks of malnutrition, a declining health status,

${ }^{1}$ The School of Nursing, Sun Yat-Sen University, Guangzhou, China. ${ }^{2}$ The Nethersole School of Nursing, Faculty of Medicine, The Chinese University of Hong Kong, Shatin, N.T., Hong Kong, Special Administrative Region, China. ${ }^{3}$ The Neighbourhood Advice-Action Council, North Point, Hong Kong, Special Administrative Region, China. ${ }^{\square}$ email: winnieso@cuhk.edu.hk 
depressive symptoms, hospital readmission, and a deteriorated quality of life ${ }^{5,6}$. Despite these drawbacks, in 2012 , nearly $29 \%$ of the 46 million community-dwelling older adults in the United States lived in single-person households, and more than half of the community-dwelling old persons ( $\geq 80$ years old) were living alone $\mathrm{e}^{4}$. In Europe, the proportion of older adults living alone has increased by $16 \%$ over the past two decades, with more than one-third of people aged $>65$ years currently living alone ${ }^{7}$. It was estimated that Chinese older population aged 65 years and above had reached 158 million in 2017 and $13.1 \%$ of them were reported to be living alone ${ }^{8}$. This number is expected to increase due to population ageing, socioeconomic development, and family nuclearisation. Older adults living alone tend to have a higher risk of developing sarcopenia than those who are not, as indicated by the results of univariate analyses in previous studies ${ }^{9}$. The sarcopenia status of older adults living alone warrants immediate attention and investigation.

The risk of sarcopenia may be more salient for Chinese older adults living alone than for those in Western communities. Because Chinese collectivist culture values family-centeredness and social cohesion, Chinese older adults can often experience stigma, discrimination, and depressive symptoms when living alone ${ }^{6,10}$. To date, several studies have reported on the prevalence of sarcopenia in the Chinese community. Liu et al. reported a prevalence of $19.3 \%$ among 4500 multi-ethnic adults aged $\geq 50$ years, according to the AWGS 2014 criteria $^{9}$. In another study, approximately $3.8 \%$ of 3997 community-dwelling older adults in Hong Kong were determined to have sarcopenia using the SARC-F questionnaire ${ }^{11}$. However, the population of Chinese older adults who live alone remains underrepresented, and there is a paucity of studies addressing the prevalence of sarcopenia and probable sarcopenia using the updated consensus criteria.

The pathophysiology of sarcopenia is multifaceted, and several factors have been investigated with respect to their role in the development of sarcopenia ${ }^{9,12,13}$. However, the reported associations of socio-demographic factors, health behaviours, and psychological factors with the risk of sarcopenia are inconsistent across studies due to heterogeneity in the study design, the operational definition of sarcopenia, the target population, and the factors analysed. Moreover, few studies have taken probable sarcopenia into account when exploring the potential determinants of sarcopenia. Identifying the associated factors, especially modifiable factors, for those at risk of sarcopenia is imperative to inform the development of therapeutic interventions tailored to mitigate sarcopenia among older adults living alone.

Therefore, the aims of the present secondary analysis were (1) to report the proportion of Chinese older adults living alone who were at risk of 'sarcopenia' and 'probable sarcopenia' using the SARC-F, CC, and SARC-CalF, according to the updated definition proposed by the AWGS 2019, and (2) to identify factors associated with an increased odds of sarcopenia.

\section{Methods}

Study design and population. This study was a secondary data analysis of a population-based crosssectional study, with the aim to investigate the nutritional status, health behaviours, lifestyle characteristics, and health status of the community-dwelling older population in Hong Kong. This study was conducted from May to September 2017. Details of the location, study population, and data collection methodology have been published elsewhere ${ }^{10}$. Briefly, the inclusion criteria were an age $>60$ years, living at home, and an ability to communicate in Chinese and provide written informed consent. Eligible participants were contacted via phone and recruited through a large registered charitable NGO. Trained interviewers conducted face-to-face interviews using a standardised questionnaire. In total, 1179 eligible older adults living alone were successfully contacted by phone, and 613 of them provided informed consent to participate in this study (response rate $=52.0 \%$ ).

Measurement. Assessment of sarcopenia. According to AWGS2019 guideline ${ }^{3}$, participants at risk of sarcopenia were identified using the SARC-F $(\geq 4), \mathrm{CC}(<34 \mathrm{~cm}$ in men, $<33 \mathrm{~cm}$ in women), or SARC-CalF $(\geq 11)$.

The SARC-F examines five domains, namely strength, assistance in walking, rising from a chair, climbing chairs, and falling episodes, and participants were asked how much difficulty they faced when performing those activities in the past year. Each item was scored from 0 (no difficulties) to 2 (a lot of difficulties) based on the participant's response. The total score was calculated by summing the participant's responses to each item, and participants with a SARC-F score $\geq 4$ were considered to have a high risk of sarcopenia. The SARC-F has been validated in a Hong Kong Chinese population and has excellent specificity and negative predictive value but a low sensitivity ${ }^{11}$.

The SARC-CalF scale is composed of six items: the first five items are identical to and scored the same as those in the SARC-F, and the sixth item is the CC (measurement of the right calf in a standing position at the point of greatest circumference). The $\mathrm{CC}$ was measured using an anthropometric measuring tape. The cut-off points for the CC are $34 \mathrm{~cm}$ for men and $33 \mathrm{~cm}$ for women ${ }^{3}$. The CC in the SARC-CalF is scored as 0 points if it is above the cut-off point and as 10 points it is below or equal to the cut-off point (suggesting low muscle mass). The SARCCalF score ranges from 0 to 20 points, and a total score of $\geq 11$ is considered to indicate a risk of sarcopenia ${ }^{14}$.

Assessment of probable sarcopenia. Probable sarcopenia was characterised by a decline in muscle strength. Handgrip strength was measured by a trained research nurse using a handheld dynamometer (TTM Smedley's Dynamometer, Japan). Each participant stood upright with the arm vertical and the dynamometer close to the body. The grip of each hand was measured, and the average value of the two handgrip measurements was presented as the maximal grip strength ${ }^{15,16}$. Weak handgrip strength (indicating probable sarcopenia) was defined as $<28 \mathrm{~kg}$ in men and $<18 \mathrm{~kg}$ in women ${ }^{3}$. 
Socio-demographic characteristics. Socio-demographic characteristics, including age, sex, marital status, educational level, employment status, monthly household income, and receipt of comprehensive social security assistance, were recorded.

Health status. Health status was determined by the Charlson comorbidity index (CCI) score and the presence of other common geriatric conditions. The participants were asked if they had ever been diagnosed with one or more of the following illnesses: myocardial infarction, angina, heart failure, peripheral vascular disease, cerebrovascular diseases, high blood pressure, dyslipidaemia, chronic lung diseases, diabetes, liver diseases, cancers, arthritis, and Parkinson's disease. The CCI represents the burden of comorbidities, and the total CCI score was calculated by summing the weighted scores of each reported condition of the individual ${ }^{17}$. Other common geriatric conditions, such as visual and hearing abilities (subjective clear, fair, and unclear) and appetite (good, normal, and poor), were rated by the participants on a 3-point Likert scale. The use of dentures and chewing difficulty were dichotomised (yes/no). The BMI was calculated as the weight in kilograms divided by the height in meters squared and categorised as underweight $\left(\leq 18.5 \mathrm{~kg} / \mathrm{m}^{2}\right)$, normal weight $\left(18.5-23.9 \mathrm{~kg} / \mathrm{m}^{2}\right)$, overweight $\left(24.0-27.9 \mathrm{~kg} / \mathrm{m}^{2}\right)$, and obese $\left(\geq 28.0 \mathrm{~kg} / \mathrm{m}^{2}\right)^{18}$.

Health behaviours and lifestyle characteristics. Health behaviours and lifestyle characteristics included the level of physical activity, dietary intake, and smoking and drinking habits.

The International Physical Activity Questionnaire Short Form (IPAQ-SF) is one of the most widely used self-report questionnaires to assess the level of physical activity. It consists of seven items and captures the daily time spent in sitting, walking, and engaging in moderate and vigorous physical activity during the last 7 days. The metabolic-equivalent minutes are calculated by multiplying the amount of time spent in the activity by 8 (for vigorous-intensity activities), 4 (for moderate-intensity activities), or 3.3 (for walking) ${ }^{19}$. The participants were categorised into low-, moderate-, and high-activity categories according to their energy expenditure per week. The Chinese IPAQ-SF has been validated against physical activity logs and has satisfactory reliability ${ }^{20}$.

The consumption of five major food groups (grains, vegetables, fruit, meat, and milk) was assessed using a culturally relevant food frequency questionnaire ${ }^{21}$. A booklet with detailed instructions, together with standard-size models of bowls, cups, and foods, were provided to illustrate the serving sizes during the interviews. Compliance with the dietary guidelines was determined by checking the dietary consumption against the recommendations of the Healthy Eating Food Pyramid for Elderly in Hong Kong.

Information on smoking (never, former smoker, or current smoker) and alcohol consumption (never, former drinker, or current drinker) statuses was directly reported by the participants.

Nutritional status. The global nutritional status of the participants was measured using the 18-item MNA. This tool assesses four aspects: (1) anthropometric conditions, including BMI, mid-arm circumference, CC, and weight loss; (2) general conditions, including lifestyle, medication, stress morbidity, and presence of neuropsychological problems; (3) dietary conditions, including number of meals, autonomy in feeding, food and fluid intake, and mode of feeding; and (4) self-perceived health-status conditions, including self-perception of health and nutritional statuses. Individuals were classified into three groups using threshold values of $<17$ for 'malnutrition', 17-23.5 for 'at risk of malnutrition', and 24-30 for 'normal nutritional status'. The MNA has been validated to screen Chinese geriatric patients for malnutrition and has satisfactory internal consistency (Cronbach's $\alpha=0.798$ ), and this instrument was suggested in previous reviews to be a useful tool for screening malnourished older adults and those at risk of malnutrition ${ }^{22}$.

Depressive symptoms. The 4-item version of the Chinese Geriatric Depression Scale (GDS-4) was used to measure depressive symptoms, with scores ranging from 0 to 4 . Participants with a score of $\geq 2$ were considered to have depressive symptoms. The GDS- 4 has a better diagnostic ability, with a sensitivity of $61 \%$ and a specificity of $81 \%$, than a comprehensive diagnostic interview ${ }^{23}$.

Data collection. Eligible participants were approached and provided with information sheets. After obtaining their written informed consent, face-to-face structured interviews were conducted by well-trained interviewers to collect participants' data. To ensure accurate assessment, all interviewers had received standardised protocol-oriented training in conducting structured interviews and anthropometric measurements before the commencement of the study.

Statistical analysis. Data analysis was performed using IBM SPSS Statistics for Windows, Version 24.0. Continuous variables are presented as the means (standard deviations) for normally distributed data. As a general of thumb, data were considered as normal if the absolute values of skewness and kurtosis are less than $2^{24}$. Categorical variables are reported as frequencies (percentages). The participants were classified as having sarcopenia risk, probable sarcopenia, or a low risk of sarcopenia according to the AWGS 2019 criteria. Sociodemographic characteristics, health status, health behaviours, lifestyle characteristics, and nutritional status were presented and compared using a one-way analysis of variance, Pearson's chi-square test, or Fisher's exact test, as appropriate. Factors with a $p$-value $<0.25$ in univariate analyses were selected for a subsequent multivariate ordinal logistic regression analysis ${ }^{25}$. This less stringent $p$-value was selected to enable a broader inclusion of potential variables to determine the contribution of each variables to sarcopenia risk, thereby yielding more accurate results in identifying the associated factors. The odds ratio, 95\% confidential interval, and $p$ value were 
reported to determine the contribution of factors to the risk of sarcopenia. Two-tailed statistical tests were conducted, and the level of significance was set at 0.05 .

Ethics approval and consent to participate. This study was conducted according to the Declaration of Helsinki and approved by the Survey and Behavioural Research Ethics Committee of the Chinese University of Hong Kong. Confidentiality of subjects' personal details was ensured by having the NGO staff contacting the eligible subjects during subject recruitment. Subjects were informed about the study details, the anonymity of any data collected, and their rights to withdraw from the study at any time through an information sheet. Subjects expressing an interest in study participation were enrolled only if they had provided written informed consent. We also obtained prior approval for using the instruments for data collection.

\section{Results}

Participant characteristics. Among the 613 participants who met the inclusion criteria for this secondary analysis, 390 participants who lived alone completed the SARC-F survey and had their CC measured. No missing data were reported. The proportion of subjects at risk of sarcopenia and those having probable sarcopenia, defined according to the AWGS 2019 criteria, was $57.7 \%$ and 30\%, respectively. The mean age of the participants was $78.1 \pm 7.4$ years. The majority of the participants were female $(58.0 \%)$, single/divorced/separated/widowed (77.7\%), and retired (95.4\%) (Table 1). The majority of the participants had only primary or lower educational attainment (73.1\%) and 20\% reported CCI score above 2. BMI assessment showed about half (57.4\%) of participants were either overweight or obese, and $7.4 \%$ were underweight. Only $12.3 \%$ of the participants reported a low level of physical activity, and nearly one-third had a Mini Nutritional Assessment (MNA) score less than 24, indicating that they were at risk of malnutrition $(27.4 \%)$ or already malnourished $(0.8 \%)$.

Factors associated with risk of sarcopenia. The results of the univariate analysis are presented in Table 2. Older age, visual and hearing impairment, difficulty in chewing food, fair appetite, and risk of malnutrition were factors associated with an increased risk of sarcopenia. However, higher educational attainment and high physical activity level were associated with decreased sarcopenia risk. Participants with a higher body mass index (BMI) had a lower risk of sarcopenia than those with a normal weight [overweight, odds ratio (OR) 0.38; $95 \%$ confidence interval (CI) $0.21,0.67 ; p=0.001$; obesity, OR $0.30 ; 95 \%$ CI $0.19,0.49 ; p<0.001$ ). In contrast, underweight participants had a higher risk of sarcopenia than participants with a normal weight (OR 5.22; $95 \%$ CI $1.17,23.41 ; p=0.031)$.

The results of a multivariate ordinal logistic regression analysis are provided in Table 3. Older age [adjusted OR (AOR) per 10-year increment: 1.68 ; $95 \%$ CI 1.17, 2.41; $p=0.005]$ and being at risk of malnutrition or malnourished (AOR 2.25; 95\% CI 1.19, 4.29; $p=0.013$ ) were significantly associated with an increased odds of having sarcopenia. Secondary school or higher educational attainment (AOR 0.50; 95\% CI 0.26, 0.96; $p=0.038$ ) and a high level of physical activity (AOR 0.36 ; $95 \%$ CI $0.16,0.86 ; p=0.020$ ) were negatively associated with the risk of developing sarcopenia. Furthermore, after controlling for confounders in our multivariate analysis, being overweight and obese remained as a factor associated with a lower risk of sarcopenia (overweight: AOR 0.39; 95\% CI, 0.20, 0.76; $p=0.006$; obesity: AOR 0.27; 95\% CI 0.15, 0.47; $p<0.001$ ).

\section{Discussion}

This secondary data analysis showed that the proportion of our cohort of Chinese community-dwelling older adults living alone who were at risk of sarcopenia and probable sarcopenia was $57.7 \%$ and $30 \%$, respectively. Sarcopenia risk was positively associated with increasing age, sedentary lifestyle, risk of malnutrition, and being malnourished, while negatively associated with educational attainment. Additionally, overweight and obese individuals were less likely to develop sarcopenia, compared with underweight individuals and those with a normal weight.

We reported that over $50 \%$ of our cohort of community-dwelling older adults living alone was at risk of sarcopenia. This proportion is much higher than that reported in a cohort study conducted in Hong Kong using the SARC-F (3.8\%) and the AWGS 2014 criteria (7.3\%), a survey conducted in China using the AWGS 2014 criteria (6.4-19.3\%), a study conducted in Singapore using dual-energy X-ray absorptiometry according to the AWGS 2019 criteria (13.6\%), and a large prospective epidemiological study (United Kingdom Biobank) using a bioimpedance analysis according to the EWGSOP2 criteria $(6.4 \%)^{9,11,12,26,27}$. This difference in sarcopenia prevalence could be partially attributed to the different diagnostic tools used in the studies. The 5-item SARC-F questionnaire is criticized by the low sensitivity (3.8-4.8\% in men and $8.2-9.9 \%$ in women), thereby precluding its ability to detect individuals who actually have the condition ${ }^{11}$. A growing body of validation studies has demonstrated that the SARC-CalF has a better diagnostic performance with improved sensitivity (60.7-83.3\%) and similar specificity (79-94.7\%) in community-dwelling older adults, compared to the use of the SARC-F $\mathrm{F}^{14}$. Incorporating the SARC-F, CC, and SARC-CalF is recommended to yield a more accurate identification of people at risk for of sarcopenia. Another possible contributor to this discrepancy could be the different ethnic groups and characteristics of the study populations. The studies conducted in western China, Singapore, and the United Kingdom recruited a considerable number of young-to-middle-aged participants, so their results may be susceptible to healthy responder bias, leading to a lower estimation of the sarcopenia prevalence ${ }^{9,12,26}$. Our findings demonstrated that sarcopenia could be common among older adults living alone thereby suggesting an urgent need to scale-up preventive and therapeutic interventions to reduce the burden of sarcopenia in this high-risk population.

The present study is among the few attempts to investigate the prevalence of probable sarcopenia according to the AWGS 2019 criteria. The prevalence of probable sarcopenia in the current study is consistent with that 
Socio-demographic characteristics

Age (years) [range: 61-106], mean (SD)

$\mathrm{Sex}^{\mathrm{a}}, \mathrm{n}(\%)$

Male

Female

Marital status, $\mathrm{n}(\%)$

Single/divorced/separated/ widowed

Married/cohabited

Educational level, $\mathrm{n}(\%)$

No formal education

Primary school

Secondary school or above

\begin{tabular}{|l|l|}
\hline $78.1(7.4)$ & $79.5(7.7)$ \\
\hline
\end{tabular}

\begin{tabular}{|l|l}
$164(42.0)$ & $93(41.3)$ \\
\hline
\end{tabular}

\begin{tabular}{l|l|l}
$226(58.0)$ & $132(58.7)$
\end{tabular}

\begin{tabular}{|l|l|}
\hline $303(77.7)$ & $172(76.4)$ \\
\hline $87(22.3)$ & $53(23.6)$
\end{tabular}

\begin{tabular}{l|l}
$87(22.3)$ & $53(23.6)$
\end{tabular}

\begin{tabular}{|l|l|}
\hline $127(32.6)$ & $87(38.7)$
\end{tabular}

\begin{tabular}{|l|l|}
\hline $158(40.5)$ & $88(39.1)$ \\
\hline
\end{tabular}

$105(26.9) \quad 50(22.2)$

Employment status, $\mathrm{n}(\%)$

Retired

Unemployed

Have part-time/full-time job

Received CSSA ${ }^{\mathrm{b}}, \mathrm{n}(\%)$

\begin{tabular}{|l}
\hline No \\
\hline
\end{tabular}

Yes

Health status

Charlson comorbidity index (CCI), mean (SD)

History of hypertension, $\mathrm{n}(\%)$

No

Yes

History of diabetes, $\mathrm{n}(\%)$

No

Yes

Visual impairment, n (\%)

No

A little

A lot

Hearing impairment, $\mathrm{n}(\%)$

Clear

Fair

Unclear

Use of denture

No

Yes

Difficulty in chewing food, $\mathrm{n}(\%)$

No

Yes

Appetite, n (\%)

Good

Normal

Bad

BMI

BMI $\left(\mathrm{kg} / \mathrm{m}^{2}\right)$

Underweight $(\leq 18.5)$

Normal (18.5-23.9)

Overweight (24.0-27.9)

Obesity ( $\geq 28.0)$

Health behaviors and lifestyle characteristics

Smoking status, $\mathrm{n}(\%)$

Non-smoker

Ex-smoker

Current smoker

Continued

\begin{tabular}{|l|l}
$372(95.4)$ & $211(94.6)$
\end{tabular}

\begin{tabular}{l|l}
$12(3.1)$ & $8(3.6)$
\end{tabular}

$6(1.6)$

4 (1.8)

\begin{tabular}{|l|l|}
\hline $144(36.9)$ & $87(38.7)$ \\
\hline $246(63.1)$ & $138(61.3)$
\end{tabular}

\begin{tabular}{|l|l|}
\hline $1.0(1.8)$ & $1.1(2.2)$ \\
\hline
\end{tabular}

\begin{tabular}{|l|l|}
\hline $144(36.9)$ & $87(38.7)$ \\
\hline $246(63.1)$ & $138(61.3)$
\end{tabular}

\begin{tabular}{|l|l}
\hline $129(33.1)$ & $69(30.7)$ \\
\hline $97(24.8)$ & $69(30.7)$
\end{tabular}

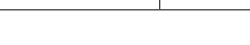

\begin{tabular}{|l|l|}
\hline $238(61.0)$ & $130(57.8)$ \\
\hline $99(25.4)$ & $56(24.9)$ \\
\hline $53(13.6)$ & $39(17.3)$
\end{tabular}

\begin{tabular}{|l|l|}
$135(34.7)$ & $78(34.8)$ \\
\hline
\end{tabular}

\begin{tabular}{l|l}
$254(65.3)$ & $146(65.2)$
\end{tabular}

\begin{tabular}{|l|l|}
\hline $239(61.3)$ & $129(57.3)$ \\
$151(38.7)$ & $96(42.9)$
\end{tabular}

\begin{tabular}{|l|l|}
\hline $219(56.2)$ & $117(52.0)$ \\
\hline $153(39.2)$ & $98(43.6)$ \\
$18(4.6)$ & $10(4.4)$ \\
$24.0(3.9)$ & $22.9(3.7)$ \\
\hline
\end{tabular}

\begin{tabular}{|l|l|}
\hline $29(7.4)$ & $27(12.0)$ \\
\hline $137(35.1)$ & $98(43.6)$ \\
\hline $75(19.2)$ & $36(16.0)$ \\
\hline $149(38.2)$ & $64(28.4)$ \\
\hline
\end{tabular}

\begin{tabular}{|l|l|}
\hline $286(73.3)$ & $168(74.7)$ \\
\hline $60(15.4)$ & $30(13.3)$ \\
\hline $44(11.3)$ & $27(12.0)$ \\
\hline
\end{tabular}

$77.0(6.7)$

$51(43.6)$

$66(56.4)$

$93(79.5)$

$24(20.5)$

$28(23.9)$

$52(44.5)$

37 (31.6)

$2(1.7)$

2 (1.7)

$44(37.6)$

$73(62.4)$

$0.9(1.4)$

$42(35.9)$

75 (64.1)

$77(65.8)$

40 (34.2)

$50(42.7)$

$41(35.0)$

$26(22.2)$

67 (57.3)

38 (32.5)

$12(10.2)$

42 (35.9)

75 (64.1)

33 (68.8)

75 (64.1)

42 (35.9)

35 (72.9)

13 (27.1)

68 (58.1)

43 (36.8)

$6(5.1)$

25.4 (3.9)

2 (1.7)

27 (23.1)

30 (25.6)

58 (49.6)

\begin{tabular}{|l|l|}
\hline $34(70.8)$ \\
$12(25.0)$ \\
$2(4.2)$ \\
$25.6(3.3)$ \\
\hline 0 \\
\hline $12(25.0)$ \\
$9(18.8)$ \\
\hline $27(56.3)$
\end{tabular}

\begin{tabular}{|l|l|}
$88(75.2)$ \\
$18(15.4)$ \\
$11(9.4)$
\end{tabular}

\begin{tabular}{|l|}
$30(62.5)$ \\
$12(25.0)$ \\
$6(12.5)$
\end{tabular}

0.046

$<0.001$

$<0.001$

$<0.001$

0.921

0.787

0.021

0.443

0.315

0.104

0.604

0.249

0.003

0.001

0.849

0.093

(18.8)

(1)




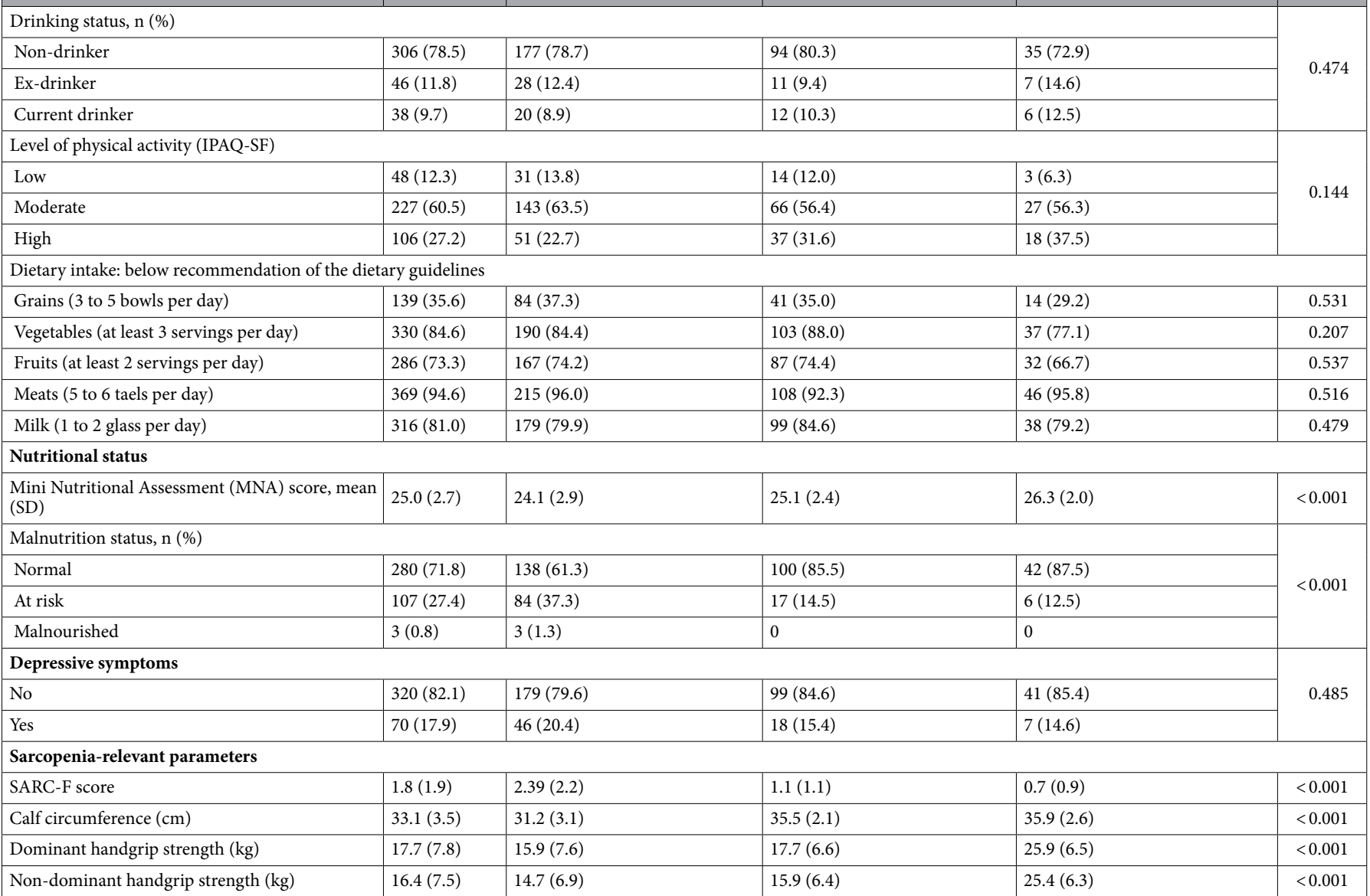

Table 1. Characteristics of the participants who live alone $(\mathrm{N}=390)$. ${ }^{\text {SSex distribution of the Hong Kong }}$ population aged 60 or above: males, 643,258 (47.6\%); females, 707,438 (52.4\%). ${ }^{\mathrm{b}}$ CSSA: comprehensive social security assistance; is a governmental financial assistance scheme providing to support individuals to meet their basic needs. ${ }^{\star}$ Continuous and categorical variables were compared among the three groups using oneway ANOVA and chi-square test respectively.

reported in a study conducted in Thailand $(28.2 \%, \mathrm{~N}=330)$ and Japan $(30.3 \%, \mathrm{~N}=552)$, but much higher than that reported in a study from Singapore $(14.0 \%, \mathrm{~N}=542)$ and in the UK Biobank study $(5.3 \%, \mathrm{~N}=499,046)^{12,15,26,28}$. Our findings suggest the usefulness of dynamometers for the easy detection of probable sarcopenia. Early detection of sarcopenia can contribute to timely referral for confirmatory diagnosis and implementation of primary preventive measures for the high-risk population.

Malnutrition, indicated by the MNA score, was found to be strongly associated with the risk of sarcopenia in the current study. The relationship between malnutrition and sarcopenia among older adults living alone is not well established, and the literature contains conflicting results on the association of the nutritional status with sarcopenia in older adults. For example, one study conducted in a geriatric outpatient population argued that the nutritional status was not associated with sarcopenia as defined according to the AWGS 2019 criteria (OR 2.16; $95 \%$ CI $0.93,4.95 ; p=0.07)^{15}$. However, Beaudart noted that malnutrition, defined according to the new European Society of Clinical Nutrition and Metabolism criteria, was significantly related to sarcopenia defined according to the EWGSOP2 criteria ${ }^{29}$. A recent prospective cohort study showed that malnourished individuals were at three-to-four-fold higher risk of developing sarcopenia compared to well-nourished individuals, during a 4-year follow-up ${ }^{29}$.

Malnutrition may contribute to the development of sarcopenia through complex pathways. In Chinese collectivist culture, eating is recognised as a social activity used to connect with friends and family. Older adults living alone are more likely to feel lonely, isolated, and depressed. These negative emotions probably reduce their appetite and dietary intake ${ }^{6}$. Poor appetite, measured by the Simplified Nutritional Appetite Questionnaire, was correlated with skeletal muscle mass index, which is recognised as an important measure of muscle quantity ${ }^{30}$. It was observed that $369(94.6 \%)$ and $316(81.0 \%)$ participants had meat and milk intakes lower than the daily recommendations of local dietary guidelines, respectively. Inadequate protein intake can limit muscle protein synthesis and increase muscle loss, thus accelerating the development of sarcopenia. Older adults who live alone tend to have fewer social relationships involving eating and are more likely to skip meals. Single-person households also face challenges in shopping for food and preparing meals, particularly in the case of individuals with 


\begin{tabular}{|c|c|c|}
\hline & OR $(95 \% \mathrm{CI})$ & $P$ value \\
\hline \multicolumn{3}{|l|}{ Socio-demographic characteristics } \\
\hline Age (per 10 years) & $1.89(1.42,2.50)$ & $<0.001$ \\
\hline \multicolumn{3}{|l|}{ Sex } \\
\hline Male (ref) & 1 & \\
\hline Female & $0.95(0.64,1.40)$ & 0.788 \\
\hline \multicolumn{3}{|l|}{ Marital status } \\
\hline Single/divorced/separated/ widowed (ref) & 1 & \\
\hline Married/cohabited & $0.85(0.53,1.37)$ & 0.508 \\
\hline \multicolumn{3}{|l|}{ Educational level } \\
\hline No formal education (ref) & 1 & \\
\hline Primary school & $0.61(0.38,0.98)$ & 0.040 \\
\hline Secondary school or above & $0.43(0.25,0.71)$ & 0.001 \\
\hline \multicolumn{3}{|l|}{ Employment status } \\
\hline Retired (ref) & 1 & \\
\hline Unemployed & $1.33(0.41,4.30)$ & 0.633 \\
\hline Have part-time/full-time job & $1.70(0.30,9.51)$ & 0.549 \\
\hline \multicolumn{3}{|l|}{ Monthly household income (HK\$) } \\
\hline$<6000$ (ref) & 1 & \\
\hline$\geq 6000$ & $3.05(0.83,11.16)$ & 0.092 \\
\hline \multicolumn{3}{|l|}{ Received CSSA ${ }^{\mathrm{a}}$} \\
\hline No (ref) & 1 & \\
\hline Yes & $1.26(0.84,1.89)$ & 0.267 \\
\hline \multicolumn{3}{|l|}{ Health status } \\
\hline Charlson comorbidity index (CCI) & $1.15(0.99,1.33)$ & 0.071 \\
\hline \multicolumn{3}{|l|}{ History of hypertension, $\mathrm{n}(\%)$} \\
\hline No (ref) & 1 & \\
\hline Yes & $0.14(0.02,0.16)$ & 0.068 \\
\hline \multicolumn{3}{|l|}{ History of diabetes, $\mathrm{n}(\%)$} \\
\hline No (ref) & 1 & \\
\hline Yes & $1.10(0.72,1.69)$ & 0.668 \\
\hline \multicolumn{3}{|l|}{ Visual impairment, n (\%) } \\
\hline No (ref) & 1 & \\
\hline A little & $1.05(0.67,1.63)$ & 0.843 \\
\hline A lot & $2.43(1.43,4.13)$ & 0.001 \\
\hline \multicolumn{3}{|l|}{ Hearing impairment, n (\%) } \\
\hline Clear (ref) & 1 & \\
\hline Fair & $1.30(0.82,2.06)$ & 0.264 \\
\hline Unclear & $2.55(1.32,4.91)$ & 0.005 \\
\hline \multicolumn{3}{|l|}{ Use of denture } \\
\hline No (ref) & 1 & \\
\hline Yes & $1.04(0.69,1.57)$ & 0.846 \\
\hline \multicolumn{3}{|l|}{ Difficulty in chewing food, n (\%) } \\
\hline No (ref) & 1 & \\
\hline Yes & $1.55(1.03,2.33)$ & 0.036 \\
\hline \multicolumn{3}{|l|}{ Appetite, n (\%) } \\
\hline Good (ref) & 1 & \\
\hline Fair & $1.63(1.08,2.47)$ & 0.020 \\
\hline Bad & $1.15(0.45,2.94)$ & 0.764 \\
\hline \multicolumn{3}{|l|}{ Body Mass Index (BMI, kg/m²) } \\
\hline Normal (ref) & 1 & \\
\hline Underweight $(\leq 18.5)$ & $5.22(1.17,23.41)$ & 0.031 \\
\hline Overweight (24.0-27.9) & $0.38(0.21,0.67)$ & 0.001 \\
\hline Obese $(\geq 28.0)$ & $0.30(0.19,0.49)$ & $<0.001$ \\
\hline \multicolumn{3}{|l|}{ Health behaviors and lifestyle characteristics } \\
\hline \multicolumn{3}{|l|}{ Smoking status } \\
\hline Non-smoker (ref) & 1 & \\
\hline Continued & & \\
\hline
\end{tabular}




\begin{tabular}{|c|c|c|}
\hline & OR $(95 \% \mathrm{CI})$ & $P$ value \\
\hline Ex-smoker & $0.64(0.38,1.09)$ & 0.097 \\
\hline Current smoker & $1.04(0.55,1.96)$ & 0.896 \\
\hline \multicolumn{3}{|l|}{ Drinking status } \\
\hline Non-drinker (ref) & 1 & \\
\hline Ex-drinker & $1.04(0.57,1.93)$ & 0.889 \\
\hline Current drinker & $0.78(0.41,1.49)$ & 0.463 \\
\hline \multicolumn{3}{|l|}{ Level of physical activity } \\
\hline Low (ref) & 1 & \\
\hline Moderate & $0.79(0.41,1.49)$ & 0.463 \\
\hline High & $0.49(0.24,0.97)$ & 0.040 \\
\hline \multicolumn{3}{|c|}{ Dietary intake: below recommendation of the dietary guidelines } \\
\hline Grains ( 3 to 5 bowls per day) & $0.82(0.54,1.23)$ & 0.337 \\
\hline Vegetables (at least 3 servings per day) & $0.91(0.53,1.55)$ & 0.730 \\
\hline Fruits (at least 2 servings per day) & $0.85(0.55,1.32)$ & 0.479 \\
\hline Meats (5 to 6 taels per day) & $0.30(0.69,1.63)$ & 0.401 \\
\hline Milk (1 to 2 glass per day) & $1.16(0.70,1.92)$ & 0.570 \\
\hline \multicolumn{3}{|l|}{ Nutritional status } \\
\hline Normal (ref) & 1 & \\
\hline At risk or malnourished & $3.82(2.29,6.35)$ & $<0.001$ \\
\hline Depressive symptoms & $0.73(0.43,1.23)$ & 0.251 \\
\hline
\end{tabular}

Table 2. Factors associated with risk of sarcopenia estimated by univariate ordinal logistic regression analysis $(\mathrm{N}=390)$. OR odds ratio, $C I$ confidence interval, ref Reference group of the categorical variable. ${ }^{a} C S S A$ Comprehensive Social Security Assistance.

sensory impairment and/or limited mobility. Hence, these individuals are less likely to prepare full, balanced meals. In our sample, $28.2 \%$ of the participants were considered malnourished or at risk of malnutrition, slightly higher than the $23.2 \%$ described for the Thai community-dwelling outpatient elderly and lower than that of the Turkish community-dwelling older population $(32.3 \%)^{31,32}$. Malnutrition can also activate the immune system and increase the synthesis of pro-inflammatory cytokines, which in turn stimulates muscle catabolism, leading to body dysfunction and an increased risk of sarcopenia ${ }^{33}$. Regular assessment and monitoring of the nutritional status of older adults living alone should therefore be prioritised as part of routine health check-ups to identify individuals at risk of sarcopenia. Integrated interventions for older adults, including advocating the intake of a high-protein diet and cultivation of social circles and addressing their psychological needs, are potentially promising approaches to optimise their nutritional status and reduce their odds of sarcopenia.

The negative association between sarcopenia and a high level of physical activity indicates that physical activity has protective effects against sarcopenia for older adults living alone. The findings in the current study concur with previous research. A longitudinal study showed that increased moderate-to-vigorous physical activity was associated with $36 \%$ protection against sarcopenia incidence after 5 years $^{34}$. A recent meta-analysis of nine studies with 2732 participants supported the protective role of physical activity against sarcopenia development (OR 0.45 , $95 \%$ CI $0.37-0.55)^{35}$. It is, however, worth noting that a considerable proportion of older population living alone who reported a moderate level of physical activity still had sarcopenia (prevalence rate of 63.5\%) and probable sarcopenia (56.4\%). This result is in line with a previous study that exclusively targeted older adults with type 2 diabetes and reported that, compared with participants engaging in a high level of physical activities, those engaging in low and moderate levels of physical activities were likely to have a higher risk of sarcopenia (low: OR 2.15; 95\% CI 1.07-4.35, $p=0.032$; moderate: OR 2.23, 95\% CI 1.07-4.66, $p=0.033)^{13}$. Moderate-intensity exercises improve muscle strength and functionality, but their effects in terms of counteracting age-related muscle loss may be limited ${ }^{36}$. These findings suggest that a high level of physical activity seems necessary to improve muscle mass and reverse sarcopenia development. As older adults living alone lack support and encouragement from an in-home caregiver, they are more vulnerable to the effects of insufficient rehabilitation. The appropriate intensity, dose, and type of rehabilitation required for older adults living alone should be comprehensively assessed.

Different approaches have been proposed for muscle mass adjustment, with the optimal estimation algorithm remaining unclear ${ }^{1}$. BMI calculated with respect to muscle mass performs better in predicting muscle strength, physical performance, daily activities, and frailty compared with BMI calculated with respect to height squared or total body mass ${ }^{37}$. In our study, compared with the participants with a normal weight, overweight and obese participants had a lower risk of sarcopenia development ( $p=0.006$ for overweight and $p<0.001$ for obese), whereas underweight participants had a higher risk compared with those having a normal weight, with this association trending towards statistical significance $(p=0.062)$. This finding is line with previous findings that suggested an obesity paradox and showed that higher BMIs are associated with better outcomes among patients with a wide variety of chronic conditions, including sarcopenia. However, opposite direction of association between BMI with functional status was found in Turkish community-dwelling male older adults ${ }^{38}$. The association between 


\begin{tabular}{|c|c|c|}
\hline & Odds ratio $(95 \% \mathrm{CI})$ & $p$-value \\
\hline \multicolumn{3}{|l|}{ Socio-demographic characteristics } \\
\hline Age per 10 years & $1.68(1.17,2.41)$ & 0.005 \\
\hline \multicolumn{3}{|l|}{ Educational level } \\
\hline No formal education (ref) & 1 & \\
\hline Primary school & $0.65(0.36,1.18)$ & 0.160 \\
\hline Secondary school or above & $0.50(0.26,0.96)$ & 0.038 \\
\hline \multicolumn{3}{|l|}{ Monthly household income (HK\$) } \\
\hline \multicolumn{3}{|l|}{$<6000$ (ref) } \\
\hline$\geq 6000$ & $1.07(0.25,4.65)$ & 0.930 \\
\hline Charlson comorbidity index (CCI) & $1.18(0.99,1.42)$ & 0.070 \\
\hline \multicolumn{3}{|l|}{ History of hypertension, $\mathrm{n}(\%)$} \\
\hline No (ref) & 1 & \\
\hline Yes & $0.69(0.41,1.15)$ & 0.153 \\
\hline \multicolumn{3}{|l|}{ Visual impairment, $\mathrm{n}(\%)$} \\
\hline No (ref) & 1 & \\
\hline A little & $0.71(0.42,1.21)$ & 0.210 \\
\hline A lot & $1.88(0.94,3.75)$ & 0.072 \\
\hline \multicolumn{3}{|l|}{ Hearing impairment, $\mathrm{n}(\%)$} \\
\hline Clear (ref) & 1 & \\
\hline Fair & $0.94(0.52,1.67)$ & 0.820 \\
\hline Unclear & $1.26(0.53,2.99)$ & 0.604 \\
\hline \multicolumn{3}{|l|}{ Difficulty in chewing food, $\mathrm{n}(\%)$} \\
\hline No (ref) & 1 & \\
\hline Yes & $1.48(0.90,2.42)$ & 0.118 \\
\hline \multicolumn{3}{|l|}{ Appetite, n (\%) } \\
\hline Good (ref) & 1 & \\
\hline Fair & $1.26(0.76,2.08)$ & 0.363 \\
\hline Bad & $0.60(0.19,1.92)$ & 0.386 \\
\hline \multicolumn{3}{|l|}{ Body Mass Index (BMI, kg/m²) } \\
\hline Normal (ref) & 1 & \\
\hline Underweight & $7.68(0.91,64.97)$ & 0.062 \\
\hline Overweight & $0.39(0.20,0.76)$ & 0.006 \\
\hline Obese & $0.27(0.15,0.47)$ & $<0.001$ \\
\hline \multicolumn{3}{|c|}{ Health behaviors and Lifestyle characteristics } \\
\hline \multicolumn{3}{|c|}{ Smoking } \\
\hline Non-smoker (ref) & 1 & \\
\hline Ex-smoker & $0.64(0.34,1.22)$ & 0.175 \\
\hline Current smoker & $0.68(0.30,1.53)$ & 0.355 \\
\hline \multicolumn{3}{|c|}{ Level of physical activity (IPAQ short form) } \\
\hline Low (ref) & 1 & \\
\hline Moderate & $0.69(0.32,1.50)$ & 0.346 \\
\hline High & $0.36(0.16,0.86)$ & 0.020 \\
\hline \multicolumn{3}{|l|}{ Nutritional status } \\
\hline Normal (ref) & 1 & \\
\hline At risk or malnourished & $2.25(1.19,4.29)$ & 0.013 \\
\hline R Square & 0.320 & \\
\hline
\end{tabular}

Table 3. Factors associated with risk of sarcopenia estimated by multivariable ordinal logistic regression analysis $(\mathrm{N}=390)$. OR odds ratio, $C I$ confidence interval, ref Reference group of the categorical variable.

excess weight and sarcopenia risk is still being debated. Increasing evidence corroborates that sarcopenic obesity is associated with accelerated functional decline, mortality, and increased risks of comorbidities, falls, disability, and long-term care placement ${ }^{3}$. The mechanism underlying the association between elevated body weight and sarcopenia risk warrants further investigation.

Multiple chronic diseases and polypharmacy significantly affect older individuals living alone, with CCI scores higher than those in the previous study ${ }^{15}$. In this study, multimorbidity was not associated with sarcopenia risk, which aligns with prior research conducted in Asia ${ }^{13,15}$. In contrast, a prospective analysis of 493,737 UK 
Biobank middle-aged and older adults demonstrated that multimorbidity was significantly associated with frailty, a condition that is interrelated with sarcopenia ${ }^{26}$. A recent multi-national population-based study that included 34,129 community-dwelling individuals aged over 50 years demonstrated that increased physical multimorbidity was associated with higher odds for weak handgrip strength. Handgrip strength is a clinically validated marker of sarcopenia risk ${ }^{16}$. Polypharmacy was also found to be associated with increased risk of sarcopenia among community-living elderly residents with type 2 diabetes in primary care clinics ${ }^{13}$. Future longitudinal studies recruiting older adults living alone from diverse settings are warranted to assess the relationship between multimorbidity and sarcopenia risk. These results have important implications for risk stratification and delivery of client-centred interventions to address the increasing challenges faced by older populations living alone.

Age and educational level were also found to be associated with the odds of sarcopenia in our study. This is in line with the findings of previous studies that suggested that older age is the most important risk factor for sarcopenia. Advancing age has been reported to be significantly associated with an increasing risk of developing sarcopenia (OR 2.28-6.87 for the 70-79 age group and 10.15-13.71 for the $\geq 80$ age group) ${ }^{15}$. The age-related pathogenesis of sarcopenia is complex and may involve interactions of genetic predisposition with changes in hormone, inflammatory markers, and myostatin expression. In our study, the participants who received secondary school or higher education were less likely to develop sarcopenia. Similar findings were reported in other surveys ${ }^{9,12}$. Individuals with higher educational attainment may be more capable of recognising personal needs and locating and utilising relevant health information, which may in turn reduce their risk of sarcopenia. Overall, our findings suggest that special attention should be paid to individuals with advancing age and lower educational attainment to mitigate their risk of sarcopenia.

Several limitations of this study deserve to be mentioned. First, this was a cross-sectional study, a design that did not allow the derivation of any causal inference between the risk of sarcopenia and associated factors; thus, reverse causality between the risk and associated factors cannot be excluded. Longitudinal studies are necessary to disentangle the directionality of the relationship observed in the current study. Furthermore, the questionnaires used in this study captured physical activity engagement (7 days) and nutritional status ( 3 months) asynchronously. However, physical activity and nutritional status may change over time, potentially affecting the accuracy of our results. Given that appetite was measured using a simple 3-point Likert scale, rather than validated questionnaires, the negative association identified between appetite and sarcopenia risk should be interpreted with caution. Additionally, residual confounding effects (e.g., functionality) are still possible and future endeavours should consider potentially relevant conditions. Gene polymorphisms have been reported to be associated with muscular strength in older men living independently ${ }^{39}$. However, this is beyond the scope of this article and warrants future investigation to specifically focus on the genetic contribution to sarcopenia and declining physical fitness. Finally, older adults with higher BMIs tended to have better outcomes. The optimal lower threshold of BMI is higher for older individuals than their younger counterparts ${ }^{38}$. Moreover, BMI cutoff values vary between ethnic groups. In this study, BMI cut-off values for the Chinese population were used, potentially limiting the generalisability of this study.

\section{Conclusion}

This study revealed that the proportion of Chinese community-dwelling older adults who live alone who were at risk of sarcopenia and those having probable sarcopenia is alarmingly high. This investigation is expected to be of great significance from a public health perspective. Age, educational level, and BMI were found to be factors associated with sarcopenia risk among older adults living alone. Maintaining a desirable nutritional status and engaging in a high level of physical activity may serve to protect or reduce the risk of developing sarcopenia. Our findings emphasise the urgent need to initiate aggressive screening and holistic lifestyle therapeutic intervention strategies for older adults living alone to mitigate the burden of sarcopenia.

\section{Data availability}

Data associated with this study can be obtained by reasonable request to the corresponding author (WKWS).

Received: 3 May 2021; Accepted: 17 September 2021

Published online: 15 November 2021

\section{References}

1. Cruz-Jentoft, A. J. et al. Sarcopenia: Revised European consensus on definition and diagnosis. Age Ageing. 48(1), 16-31 (2019).

2. Bahat, G. \& İlhan, B. Sarcopenia and the cardiometabolic syndrome: A narrative review. Euro. Geriatr. Med. 7(3), 220-223 (2016).

3. Chen, L. K. et al. Asian working group for Sarcopenia: 2019 Consensus update on sarcopenia diagnosis and treatment. J. Am. Med. Dir. Assoc. 21(3), 300-307 e302 (2020).

4. Lee, S. M. \& Edmonston, B. Living alone among older adults in canada and the U.S.. Healthcare 7(2), 68 (2019).

5. Chan, H. Y. L. et al. Undernutrition risk and recent hospital admission in older adults living alone or with spouse only. Arch. Gerontol. Geriatr. 94, 104351 (2021).

6. So, W. K. W. et al. Risks of malnutrition and depression in older adults living alone or with a spouse in Hong Kong. Psychogeriatrics 20(6), 936-940 (2020).

7. Pleschberger, S., Reitinger, E., Trukeschitz, B. \& Wosko, P. Older people living alone (OPLA): Non-kin-carers' support towards the end of life: Qualitative longitudinal study protocol. BMC Geriatr. 19(1), 219 (2019).

8. Han, Y. et al. Aging in China: Perspectives on public health. Global Health J. 4(1), 11-17 (2020).

9. Liu, X. et al. Prevalence of sarcopenia in multi ethnics adults and the association with cognitive impairment: Findings from WestChina health and aging trend study. BMC Geriatr. 20(1), 63 (2020).

10. Wong, M. M. H. et al. Malnutrition risks and their associated factors among home-living older Chinese adults in Hong Kong: Hidden problems in an affluent Chinese community. BMC Geriatr. 19(1), 138 (2019).

11. Woo, J., Leung, J. \& Morley, J. E. Validating the SARC-F: A suitable community screening tool for sarcopenia?. J. Am. Med. Dir. Assoc. 15(9), 630-634 (2014). 
12. Pang, B. W. J. et al. Prevalence and associated factors of Sarcopenia in Singaporean adults-the yishun study. J. Am. Med. Dir. Assoc. 22(4), 885 e881-885 e810 (2021).

13. Sazlina, S.-G., Lee, P. Y., Chan, Y. M., Hamid, M. S. \& Tan, N. C. The prevalence and factors associated with sarcopenia among community living elderly with type 2 diabetes mellitus in primary care clinics in Malaysia. PLoS ONE 15(5), e0233299 (2020).

14. Yang, M. et al. Screening sarcopenia in community-dwelling older adults: SARC-F vs SARC-F combined with calf circumference (SARC-CalF). J. Am. Med. Dir. Assoc. 19(3), 277 e271-277 e278 (2018).

15. Therakomen, V., Petchlorlian, A. \& Lakananurak, N. Prevalence and risk factors of primary sarcopenia in community-dwelling outpatient elderly: A cross-sectional study. Sci. Rep. 10(1), 19551 (2020).

16. Vancampfort, D., Stubbs, B., Firth, J. \& Koyanagi, A. Handgrip strength, chronic physical conditions and physical multimorbidity in middle-aged and older adults in six low- and middle income countries. Eur. J. Int. Med. 61, 96-102 (2019).

17. Quan, H. et al. Updating and validating the Charlson comorbidity index and score for risk adjustment in hospital discharge abstracts using data from 6 countries. Am. J. Epidemiol. 173(6), 676-682 (2011).

18. Gao, M. et al. The cut-off points of body mass index and waist circumference for predicting metabolic risk factors in Chinese adults. Zhonghua Liu Xing Bing Xue Za Zhi 40(12), 1533-1540 (2019).

19. Forde, C. Scoring the International Physical Activity Questionnaire (IPAQ). Accessed 2021.

20. Macfarlane, D. J., Lee, C. C., Ho, E. Y., Chan, K. L. \& Chan, D. T. Reliability and validity of the Chinese version of IPAQ (short, last 7 days). J. Sci. Med. Sport. 10(1), 45-51 (2007).

21. Health HKDo. Health Eating Food Pyramid for Elderly. . Accessed.

22. Han, Y., Li, S. \& Zheng, Y. Predictors of nutritional status among community-dwelling older adults in Wuhan, China. Public Health Nutr. 12(8), 1189-1196 (2009).

23. D’Ath, P., Katona, P., Mullan, E., Evans, S. \& Katona, C. Screening, detection and management of depression in elderly primary care attenders: I: The acceptability and performance of the 15 item Geriatric Depression Scale (GDS15) and the development of short versions. Fam. Pract. 11(3), 260-266 (1994).

24. Fagerland, M. W. t-tests, non-parametric tests, and large studies-A paradox of statistical practice?. BMC Med. Res. Methodol. 12(1), 78 (2012).

25. Bursac, Z., Gauss, C. H., Williams, D. K. \& Hosmer, D. W. Purposeful selection of variables in logistic regression. Sour. Code Biol. Med. 3, 17 (2008).

26. Dodds, R. M., Granic, A., Robinson, S. M. \& Sayer, A. A. Sarcopenia, long-term conditions, and multimorbidity: Findings from UK Biobank participants. J. Cachexia Sarcopenia Muscle. 11(1), 62-68 (2020).

27. Han, P. et al. Prevalence and factors associated with Sarcopenia in suburb-dwelling older Chinese using the Asian working group for Sarcopenia definition. J. Gerontol. A Biol. Sci. Med. Sci. 71(4), 529-535 (2016).

28. Kurose, S. et al. Prevalence and risk factors of sarcopenia in community-dwelling older adults visiting regional medical institutions from the Kadoma Sarcopenia Study. Sci. Rep. 10(1), 19129 (2020).

29. Beaudart, C. et al. Malnutrition as a strong predictor of the onset of sarcopenia. Nutrients 11(12), 2883 (2019).

30. İlhan, B., Bahat, G., Erdoğan, T., Kılıç, C. \& Karan, M. A. Anorexia is independently associated with decreased muscle mass and strength in community dwelling older adults. J. Nutr. Health Aging. 23(2), 202-206 (2019).

31. Bahat, G. et al. Assessments of functional status, comorbidities, polypharmacy, nutritional status and sarcopenia in Turkish community-dwelling male elderly. Aging Male. 16(2), 67-72 (2013).

32. Bahat, G. et al. Comorbidities, polypharmacy, functionality and nutritional status in Turkish community-dwelling female elderly. Aging Clin. Exp. Res. 26(3), 255-259 (2014).

33. Robinson, S. M. et al. Does nutrition play a role in the prevention and management of sarcopenia?. Clin. Nutr. 37(4), 1121-1132 (2018).

34. Mijnarends, D. M. et al. Physical activity and incidence of sarcopenia: The population-based AGES-Reykjavik Study. Age Ageing. 45(5), 614-620 (2016).

35. Steffl, M. et al. Relationship between sarcopenia and physical activity in older people: A systematic review and meta-analysis. Clin. Interv. Aging. 12, 835-845 (2017).

36. Rosique-Esteban, N. et al. Leisure-time physical activity at moderate and high intensity is associated with parameters of body composition, muscle strength and sarcopenia in aged adults with obesity and metabolic syndrome from the PREDIMED-Plus study. Clin. Nutr. 38(3), 1324-1331 (2019).

37. Bahat, G., Kilic, C., Ilhan, B., Karan, M. A. \& Cruz-Jentoft, A. Association of different bioimpedanciometry estimations of muscle mass with functional measures. Geriatr. Gerontol. Int. 19(7), 593-597 (2019).

38. Bahat, G. et al. Body mass index and functional status in community dwelling older Turkish males. Aging Male. 18(4), 228-232 (2015).

39. Bahat, G. et al. BsmI polymorphism in the vitamin D receptor gene is associated with leg extensor muscle strength in elderly men. Aging Clin. Exp. Res. 22(3), 198-205 (2010).

\section{Acknowledgements}

The authors gratefully acknowledge the Neighbourhood Advice-Action Council for their funding support and coordination. We would like to thank all the study participants of their participation in our study.

\section{Author contributions}

L.C. contributed to the data collection, analysis, and interpretation, and drafted the manuscript. J.W.H.S. contributed to the study conception and design, study coordination, and data interpretation. H.Y.L.C. contributed to the study conception and design, data interpretation. K.C.C. contributed to study design, data analysis and interpretation. R.K.Y.C. contributed to the study coordination and data collection. M.M.H.W. contributed to the study coordination and data collection. F.Y.K.L. contributed to the study coordination and data collection. T.Y.L. contributed to the study coordination and data collection. E.S.M.F. contributed to the study coordination and data collection. K.M.T. contributed to the study coordination and data collection. W.K.W.S. contributed to the study conception, design, coordination, and data interpretation. All authors critically revised the manuscript and approved the final version.

\section{Competing interests}

The authors declare no competing interests.

\section{Additional information}

Correspondence and requests for materials should be addressed to W.K.W.S. 
Reprints and permissions information is available at www.nature.com/reprints.

Publisher's note Springer Nature remains neutral with regard to jurisdictional claims in published maps and institutional affiliations.

(c) (i) Open Access This article is licensed under a Creative Commons Attribution 4.0 International License, which permits use, sharing, adaptation, distribution and reproduction in any medium or format, as long as you give appropriate credit to the original author(s) and the source, provide a link to the Creative Commons licence, and indicate if changes were made. The images or other third party material in this article are included in the article's Creative Commons licence, unless indicated otherwise in a credit line to the material. If material is not included in the article's Creative Commons licence and your intended use is not permitted by statutory regulation or exceeds the permitted use, you will need to obtain permission directly from the copyright holder. To view a copy of this licence, visit http://creativecommons.org/licenses/by/4.0/.

(C) The Author(s) 2021, corrected publication 2022 